\title{
Impacto clínico-funcional del traslado directo desde domicilio a un centro con servicio de hemodinamia en el infarto agudo de miocardio con elevación de ST
}

\author{
Dres. Lía Sapriza, Leandro Jubany, Julia Aramburu, Daniela Korytnicki, \\ Marcos Pouso, Natalia Lluberas, Gabriel Parma, Sebastián Lluberas, \\ Carolina Artucio, Andrés Tuzman, Ricardo Lluberas, Daniel Mallo
}

\begin{abstract}
Resumen
Antecedentes: la demora en la reperfusión del infarto agudo de miocardio con elevación del ST (IAMCST) es un determinante mayor de su evolución clínica y funcional.

Objetivo: analizar el impacto del traslado directo desde domicilio a un centro con hemodinamia sobre los tiempos de reperfusión y la evolución clínico-ecocardiográfica del IAMCST.

Material y método: se diseñó un estudio prospectivo, observacional, que incluyó los pacientes con IAMCST recibidos en el servicio de hemodinamia del Instituto de Cardiología Intervencionista de Casa de Galicia (INCI) para angioplastia transluminal coronaria (ATC) primaria del 1ํ de febrero de 2016 al 30 de setiembre de 2016. Los pacientes se clasificaron en dos grupos: 1) traslado directo desde domicilio a servicio de hemodinamia y 2) traslado desde otro centro asistencial. Se evaluaron los tiempos dolor-primer contacto médico (PCM) y PCM-balón. Se comparó la evolución clínica, la fracción de eyección del ventrículo izquierdo (FEVI) y el score de contractilidad sectorial del VI a corto plazo (a las 48 horas tras el ingreso y al mes) entre ambos grupos.

Resultados: se incluyeron 124 pacientes, 38 mujeres (31\%), edad media 63,2 $\pm 13,5$ años. El tiempo PCM-balón representó el 54,7\% del tiempo isquémico. Provenían de domicilio 51 pacientes (41\%). El tiempo de reperfusión en el grupo 1 fue $284 \pm 241$ min vs $498 \pm 309$ min en el grupo 2 ( $p<0,001$ ), mientras que el tiempo PCM-balón del grupo 1 fue 111 $\pm 76,3$ min vs $263 \pm 175,1$ min del grupo 2 , ( $<<0,001$ ). No existieron diferencias significativas entre los grupos 1 y 2 en referencia a la FEVI medida al ingreso $(49,5 \pm 9,33 \%$ vs $46,5 \pm 9,78 \%)$ y al mes $(53,0 \pm-8,5)$ vs $(50,2 \pm 10,5)$. El score de contractilidad inicial fue menor en el grupo $1(1,37 \pm 0,39)$ que en el grupo $2(1,46 \pm 0,31)(p=0,029)$, mientras que no mostró diferencias significativas en el control al mes $(1,23 \pm 0,26)$ vs $1,34 \pm 0,32$. La mortalidad total fue de 12 pacientes $(9,7 \%)$ y antes de las 48 horas, 8 pacientes $(6,5 \%)$. La tasa de eventos cardíacos adversos mayores (ECAM: reinfarto, revascularización urgente, muerte y accidente cerebrovascular [ACV]) no difirió entre ambos grupos. Los pacientes que sufrieron ECAM presentaron mayor score de contractilidad inicial y menor FEVI inicial y al mes.

Conclusión: la estrategia de traslado directo desde domicilio a un centro de hemodinamia se asocia con un menor tiempo isquémico total a expensas de un menor tiempo PCM-balón, menor tiempo PCM-puerta y con un mejor score de contractilidad segmentaria inicial.
\end{abstract}

Palabras clave: $\quad$ INFARTO DEL MIOCARDIO REPERFUSIÓN MIOCÁRDICA

TRASLADO DE PACIENTES

ANGIOPLASTIA

Instituto de Cardiología Intervencionista INCI. Centro Cardiovascular Casa de Galicia, Montevideo, Uruguay. 


\title{
Clinical-functional impact of direct transfer from home to a center with hemodynamic service in acute myocardial infarction with ST elevation
}

\author{
Summary \\ Background: delayed reperfusion of acute myocardial infarction with ST elevation (STEMI) is a major determinant of its \\ clinical and functional course. \\ Objective: to analyze the impact of the direct transfer from home to a center with hemodynamic service on the reperfusion \\ times and in the clinical and echocardiographic evolution of the STEMI. \\ Method: a prospective, observational study was designed that included patients with STEMI received at the INCI hemody- \\ namic service for primary coronary transluminal angioplasty (TCA) from 1st.February 2016 to 30th September 2016. Pa- \\ tients were classified in two groups: 1) direct transfer from home to hemodynamic service and 2) transfer from another care \\ center. Pain-first medical contact (FMC) and FMC-device times were evaluated. The short-term clinical evolution, the left \\ ventricular ejection fraction (LVEF) and left ventricular sector contractility score (at 48 hours post admission and at one \\ month) were compared between both groups. \\ Results: we included 124 patients, 38 (31\%) women, mean age $63.2 \pm 13.5$ years. FMC-device time accounted for $54.7 \%$ of is- \\ chemic time. 51 patients (41\%) were direct transfer from domicile. The reperfusion time in group 1 was $284 \pm 241$ min vs. 498 \\ \pm 309 min in group $2(\mathrm{p}<0.001)$, while the FMC-device time of group 1 was $111 \pm 76.3 \mathrm{~min}$ vs $263 \pm 175.1 \mathrm{~min}$ of group 2 , (p \\ $<0.001)$. There were no significant differences between groups 1 and 2 in relation to LVEF measured at admission ( $49.5 \pm$ \\ $9.33 \%$ vs. $46.5 \pm 9.78 \%)$ and at one month (53.0 \pm-8.5$)$ vs. $(50.2 \pm 10.5)$. The initial contractility score was lower in group 1 \\ $(1.37 \pm 0.39)$ than in group $2(1.46 \pm 0.31)(\mathrm{p}=0.029)$, whereas it did not show significant differences in control and at one \\ month $(1.23 \pm 0.26) .1 .34 \pm 0.32$. The total mortality was 12 patients $(9.7 \%)$ and 8 patients $(6.5 \%)$ before 48 hours. The rate of \\ major adverse cardiac events (MACE) did not differ between the two groups. \\ Conclusion: the strategy of direct transfer from home to a hemodynamic center is associated with a shorter total ischemic time \\ at the expense of a shorter FMC-device time and a shorter FMC-door time and with a better segmental contractility score. \\ Key words:

MYOCARDIAL INFARCTION
MYOCARDIAL REPERFUSION
PATIENTS TRANSFER
ANGIOPLASTY

\section{Antecedentes}

El tiempo de retraso en la reperfusión es un determinante pronóstico mayor de la evolución clínica y ecocardiográfica en los pacientes con $\operatorname{IAMCST}^{(1-3)}$. Durante las primeras semanas que siguen a la reperfusión existe una mejoría de la contractilidad en las zonas de miocardio atontado ${ }^{(4,5)}$. La ATC primaria se ha impuesto como la opción de reperfusión de elección siempre que se acceda a la misma de forma precoz, recomendándose por sobre los agentes fibrinolíticos cuando el tiempo PCM-balón no supera los 120 minutos $^{(6-10)}$.

Existen importantes experiencias a nivel internacional que han demostrado ser eficaces en disminuir el tiempo PCM-balón en el IAMCST ${ }^{(11-15)}$. En Uruguay, la ATC primaria es una estrategia de reperfusión del IAMCST eficaz y segura, con mortalidad inicial y alejada que se asimila a lo reportado internacionalmente ${ }^{(16)}$. El registro RENATIA evidenció una demora de 255 minutos desde el inicio de los síntomas hasta la reperfusión con ATC primaria; en el $50 \%$ de estos pacientes se requería el traslado desde otro centro asistencial a un centro con servicio de hemodinamia $^{(17)}$. Estos hallazgos locales subrayan la importancia de la organización de los sistemas de respuesta al IAMCST para disminuir el tiempo isquémico.

\section{Principales aportes}

- En Uruguay existe importante retraso en el acceso a la ATC primaria en pacientes con IAMCEST.Del tiempo isquémico total, un $45,3 \%$ corresponde al tiempo dolor-PCM y el resto a demoras del sistema de salud.

- El traslado directo desde domicilio a centro con hemodinamia disminuye el tiempo PCM-balón en 58\% y el tiempo isquémico total en $43 \%$.

- Los pacientes con traslado directo presentaron tiempos PCM-balón en los rangos exigidos por las recomendaciones actuales.

- Aquellos que sufrieron ECAM presentaron mayor score de contractilidad inicial y menor FEVI inicial y al mes.

\section{Objetivo}

Analizar el impacto del traslado directo desde domicilio a un centro con hemodinamia sobre los tiempos de reperfusión y la evolución clínica y ecocardiográfica del IAMCST.

\section{Material y método}

Se realizó un estudio prospectivo observacional que 
incluyó a todos los pacientes con IAMCST de menos de 24 horas de evolución recibidos en el centro INCI para ATC primaria desde el $1^{\circ}$ de febrero de 2016 al 30 de setiembre de 2016 . Se excluyeron los pacientes con antecedente de IAM o revascularización previa y los portadores de valvulopatía mitral o aórtica severas. Se volcaron los datos en una base que incluyó los tiempos de reperfusión, variables clínicas y angiográficas (topografía del infarto, clasificación de Killip y Kimball, aspiración trombótica endovascular, tipo de stent implantado, score de flujo TIMI) así como ecocardiográficas. Los pacientes se clasificaron en dos grupos: 1) pacientes que recibieron traslado directo desde el domicilio a un centro con servicio de hemodinamia a través de una unidad de emergencia móvil (UEM) y los que consultaron directamente por sus propios medios en la emergencia de un centro con hemodinamia, y 2) pacientes valorados inicialmente en domicilo por UEM que fueron trasladados a un centro sin hemodinamia o que consultaron en emergencia de un centro sin hemodinamia, para desde allí ser trasladados a otro centro con hemodinamia. Se evaluaron los tiempos: isquémico total (desde el inicio de los síntomas hasta la reperfusión), dolor-PCM, PCM-balón y puerta-balón. Se analizaron las características clínicas de los pacientes al momento de la presentación, durante la evolución hospitalaria y al mes. Se registraron los ECAM (muerte, reinfarto, necesidad de nueva revascularización urgente y $\mathrm{ACV}$ ) en la internación y mediante un control ambulatorio en policlínica al mes. Aquellos que no concurrieron al control clínico mensual fueron contactados telefónicamente. Se realizó ecocardiograma transtorácico (ETT) entre las 24 y 48 horas del cuadro inicial y al mes del procedimiento. Los ecocardiogramas fueron realizados con las vistas estándar (paraesternal ejes largo y corto, apical 4, 3 y 2 cámaras). Las imágenes fueron capturadas para su posterior análisis por dos operadores independientes que no tenían acceso a la historia clínica ni a los resultados del ETT previo. Se calculó la FEVI a partir de los volúmenes de fin de diástole y fin de sístole obtenidos por método biplano de discos (Simpson modificado). La motilidad segmentaria se analizó utilizando un modelo de 16 segmentos, asignando 4 grados de score de contractilidad a cada segmento (donde $1=$ contractilidad normal, $2=$ hipoquinesia, $3=$ aquinesia, $4=$ disquinesia). Posteriormente se calculó el score de contractilidad segmentaria del VI mediante el cociente entre la suma del score de cada segmento y el número de segmentos.

\section{Análisis estadístico}

Las variables continuas se compararon mediante la prueba U de Mann Whitney entre grupos ya que en ningún caso las distribuciones son normales (test de Kolgomorov-Smirnov con corrección de Lilliefors, $p=0,000$ para todas las variables consideradas) y test de Wilcoxon de los rangos de los signos entre valores ecocardiográficos iniciales y finales en cada grupo. Las variables categóricas se compararon mediante test exacto de Fisher. En los análisis se consideró como nivel de significación un valor de $\mathrm{p}<0,05$ (dos colas).

\section{Resultados}

Se incluyeron 124 pacientes, 38 (31\%) mujeres. La edad media \pm DE fue de $63,2 \pm 13,5$ años. Las características de la población de estudio se muestran en la tabla 1 .

\section{Análisis del tiempo isquémico}

La media $\pm \mathrm{DE}$ del tiempo isquémico total fue $410 \pm 301 \mathrm{~min}$, con una mediana de $300 \mathrm{~min}$ (60-1.440 min). El tiempo dolor-PCM tuvo una media \pm DE de 209 \pm 241 min y una mediana de 120 min (15-1.080 $\mathrm{min})$. El tiempo dolor-PCM corresponde a $45,3 \%$ del tiempo isquémico total, valor obtenido promediando el porcentaje al que corresponde este tiempo para cada paciente. El tiempo medio \pm DE PCM-balón fue 201 161 min, con una mediana de $150 \mathrm{~min}$ (30-900 $\mathrm{min}$ ), lo que corresponde a $54,7 \%$ del tiempo total. El tiempo medio puerta de centro con servicio de hemodinamia-balón fue $53 \pm 50$, con una mediana de $45 \mathrm{~min}$ ( $15-480 \mathrm{~min}$ ), lo que constituye el 18,4\% del tiempo isquémico total. En la tabla 2 y la figura 1 se caracterizan los tiempos en toda la población de estudio y en ambos grupos.

\section{Traslados en ambos grupos}

Provenían de domicilio (grupo 1): 51 pacientes (41\%) y de otro centro asistencial (grupo 2): 73 pacientes (59\%). Cinco pacientes del grupo 1 (9,8\%) consultaron por sus medios en la emergencia de un centro con servicio de hemodinamia, mientras que $46(90,2 \%)$ fueron trasladados desde su domicilio por la UEM. Veinte pacientes del grupo $2(27,4 \%)$ consultaron por sus medios en emergencia de centro sin hemodinamia y $53(72,6 \%)$ fueron trasladados por UEM desde su domicilio a un centro sin hemodinamia, para luego requerir un segundo traslado hacia el centro con hemodinamia.

\section{Análisis temporal según grupos (figura 2, tabla 2)}

La media \pm DE de tiempo de reperfusión del grupo 1 fue $284 \pm 241 \mathrm{~min}$, con una mediana de $240 \mathrm{~min}$ (60-1200), mientras que en el grupo 2 fue de $498 \pm 309 \mathrm{~min}$, con una mediana de $375 \mathrm{~min}$ 


\begin{tabular}{|c|c|c|c|c|}
\hline & Población total & Grupo 1 & Grupo 2 & \\
\hline $\mathrm{N}$ & $124(100 \%)$ & $51(41 \%)$ & $73(59 \%)$ & \\
\hline Edad (años) & $63,2 \pm 13,5$ & $62,9 \pm 12,3$ & $63,5 \pm 14,7$ & $\mathrm{~ns}$ \\
\hline Sexo F & $38(31 \%)$ & $14(27 \%)$ & $24(33 \%)$ & $\mathrm{ns}$ \\
\hline HTA & $72(58 \%)$ & $29(57 \%)$ & $43(59 \%)$ & $\mathrm{ns}$ \\
\hline $\mathrm{DM}$ & $31(25 \%)$ & $13(25 \%)$ & $18(25 \%)$ & $\mathrm{ns}$ \\
\hline Dislipemia & $31(25 \%)$ & $13(25 \%)$ & $18(25 \%)$ & $\mathrm{ns}$ \\
\hline Tabaquismo & $70(56 \%)$ & $29(57 \%)$ & $41(56 \%)$ & $\mathrm{ns}$ \\
\hline Obesidad & $34(27 \%)$ & $15(29 \%)$ & $19(26 \%)$ & $\mathrm{ns}$ \\
\hline IR & $16(13 \%)$ & $6(12 \%)$ & $10(14 \%)$ & $\mathrm{ns}$ \\
\hline \multicolumn{5}{|l|}{ Topografía } \\
\hline Anterior & $45(36 \%)$ & $17(33 \%)$ & $28(38 \%)$ & $\mathrm{ns}$ \\
\hline Inferior & $74(60 \%)$ & $32(63 \%)$ & $42(58 \%)$ & $\mathrm{ns}$ \\
\hline Lateral & $5(4 \%)$ & $2(4 \%)$ & $3(4 \%)$ & $\mathrm{ns}$ \\
\hline \multicolumn{5}{|l|}{ KK } \\
\hline I & $95(77 \%)$ & $44(86 \%)$ & $51(70 \%)$ & ns \\
\hline II & $18(15 \%)$ & $3(6 \%)$ & $15(20 \%)$ & $\mathrm{p}=0,037$ \\
\hline III & $3(2 \%)$ & $1(2 \%)$ & $2(3 \%)$ & $\mathrm{ns}$ \\
\hline IV & $8(6 \%)$ & $3(6 \%)$ & $5(7 \%)$ & $\mathrm{ns}$ \\
\hline Tromboaspiración & $37(30 \%)$ & $17(33 \%)$ & $20(27 \%)$ & $\mathrm{ns}$ \\
\hline BMS & $82(66 \%)$ & $31(61 \%)$ & $51(70 \%)$ & $\mathrm{ns}$ \\
\hline DES & $42(34 \%)$ & $20(39 \%)$ & $22(30 \%)$ & $\mathrm{ns}$ \\
\hline \multicolumn{5}{|l|}{ TIMI } \\
\hline 0 & $4(3 \%)$ & $2(4 \%)$ & $2(3 \%)$ & ns \\
\hline 1 & $3(2 \%)$ & $2(4 \%)$ & $1(1 \%)$ & $\mathrm{ns}$ \\
\hline 2 & $8(6 \%)$ & $3(6 \%)$ & $5(7 \%)$ & $\mathrm{ns}$ \\
\hline 3 & $109(88 \%)$ & $44(86 \%)$ & $65(89 \%)$ & $\mathrm{ns}$ \\
\hline
\end{tabular}

(90-1.440 min) ( $\mathrm{p}<0,001)$. El tiempo PCM-balón del grupo 1 fue $111 \pm 76 \mathrm{~min}$, mediana=92 (30-480 min); para el grupo 2 la media (DE) fue 263 $\min \pm 175 \min$ y la mediana fue de $192 \min (60-900$ $\min )(\mathrm{p}<0,001)$.

\section{Evaluación ecocardiográfica}

Se realizó ETT inicial en 116 pacientes, ya que de los 124 pacientes incluidos, 8 fallecieron previo a la realización del mismo, lo cual arroja una mortalidad hospitalaria de 6,5\%. El tiempo de seguimiento fue de un mes, con una mortalidad total de 9,7\% $(\mathrm{n}=12)$. De los 112 pacientes vivos al mes, todos se contactaron para control clínico, 16 controles fueron telefónicos y 96 en consultorio. De estos 96 controles clínicos presenciales se excluyeron 11 pacientes del análisis ecocardiográfico por haber recibido nueva revascularización con ATC o cirugía, por lo que fueron analizados 85 ecocardiogramas al mes del IAMCST.

La FEVI inicial fue de 49,5 $\pm 9,33 \%$ en el grupo 1 $\mathrm{y}$ de $46,5 \pm 9,7 \%$ en el grupo $2(\mathrm{p}=0,114)$. La FEVI al mes fue de $53 \pm 8,5 \%$ en el grupo 1 y de $50,2 \pm 10,5 \%$ en el grupo $2(p=0,309)$. El score de contractilidad 


\begin{tabular}{|c|c|c|c|}
\hline & Población total & Grupo 1 & Grupo 2 \\
\hline \multicolumn{4}{|l|}{ Tiempo isquémico total } \\
\hline Media $\pm \mathrm{DE}$ & $410 \pm 301$ & $284 \pm 241$ & $498 \pm 309$ \\
\hline Mediana (mínimo-máximo) & $300(60-1440)$ & $240(60-1200)$ & $375(90-1440)$ \\
\hline \multicolumn{4}{|l|}{ Tiempo dolor-PCM } \\
\hline Media & $209 \pm 241$ & $172 \pm 219$ & $234 \pm 254$ \\
\hline Mediana & $120(15-1080)$ & $85(15-1080)$ & $120(15-1080)$ \\
\hline \multicolumn{4}{|l|}{ Tiempo PCM-balón } \\
\hline Media & $201 \pm 161$ & $111 \pm 76$ & $263 \pm 175$ \\
\hline Mediana & $150(30-900)$ & $92(30-480)$ & $192(60-900)$ \\
\hline \multicolumn{4}{|l|}{ Tiempo puerta-balón } \\
\hline Media & $53 \pm 50$ & $44 \pm 32$ & $59 \pm 58$ \\
\hline Mediana & $45(15-480)$ & $30(15-210)$ & $47(15-480)$ \\
\hline
\end{tabular}

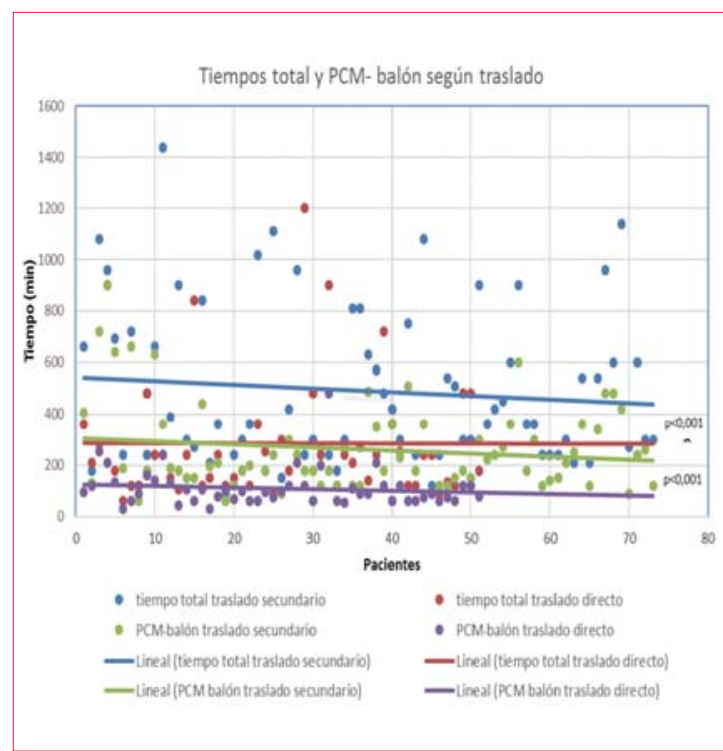

Figura 1. Gráfico de dispersión de los diferentes tiempos analizados para cada paciente según grupos y las medianas de estos tiempos en líneas. PCM: primer contacto médico

inicial fue de $1,37 \pm-0,39$ en el grupo 1 y $1,46 \pm 0,31$ en el grupo 2, $(\mathrm{p}=0,029)$. El score de contractilidad obtenido al mes fue de $1,23 \pm 0,26$ para el grupo $1 \mathrm{y}$ $1,34 \pm 0,32$ para el grupo 2 ( $\mathrm{p}=0,124)$, lo que se representa en la figura 3.

En toda la población hubo mejoría significativa de la FEVI al mes: $3,21 \pm 6,22 \%$, mediana de $2 \%$ $(\mathrm{p}<0,001)$ y del score de contractilidad: mediana de $-0,0625(\mathrm{p}<0,001)$. No hubo diferencias significati-

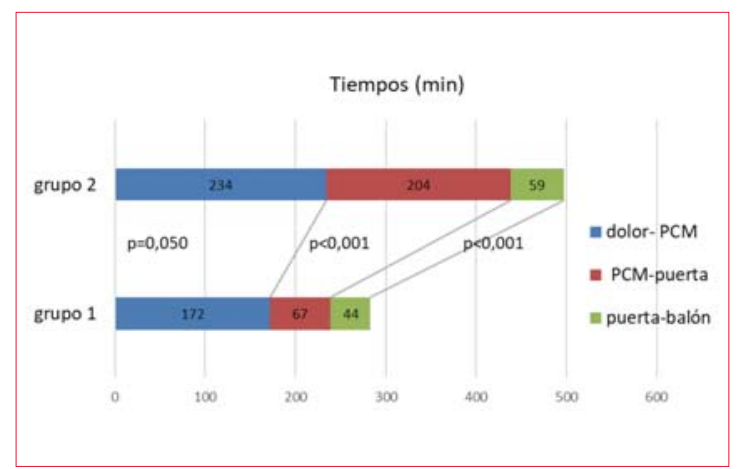

Figura 2. Se muestra la reducción de cada uno de los tiempos analizados, que determina una disminución del tiempo isquémico total para el grupo 1 (con traslado directo) respecto al grupo 2 (traslado secundario). PCM: primer contacto médico.

vas en la mejoría de FEVI ni en el score de contractilidad entre ambos grupos

\section{Tasa de eventos y variables ecocardiográficas}

La mortalidad al mes fue de 7,8\% $(\mathrm{n}=7)$ en el grupo 1 y $10,9 \%(n=8)$ en el grupo $2(\mathrm{NS})$. Once pacientes sufrieron ECAM al mes en el grupo $1(21,6 \%)$ y 12 en el grupo $2(16,4 \%)$, sin diferencias significativas. En la figura 4 se analiza la tasa de eventos al mes en cada grupo.

Si evaluamos los pacientes con ECAM se observa que tuvieron una FEVI inicial de $40,6 \pm 7,7 \%$ en comparación con $48,4 \pm 9,6 \%$ en los pacientes libres de eventos $(\mathrm{p}=0,008)$. Asimismo, 


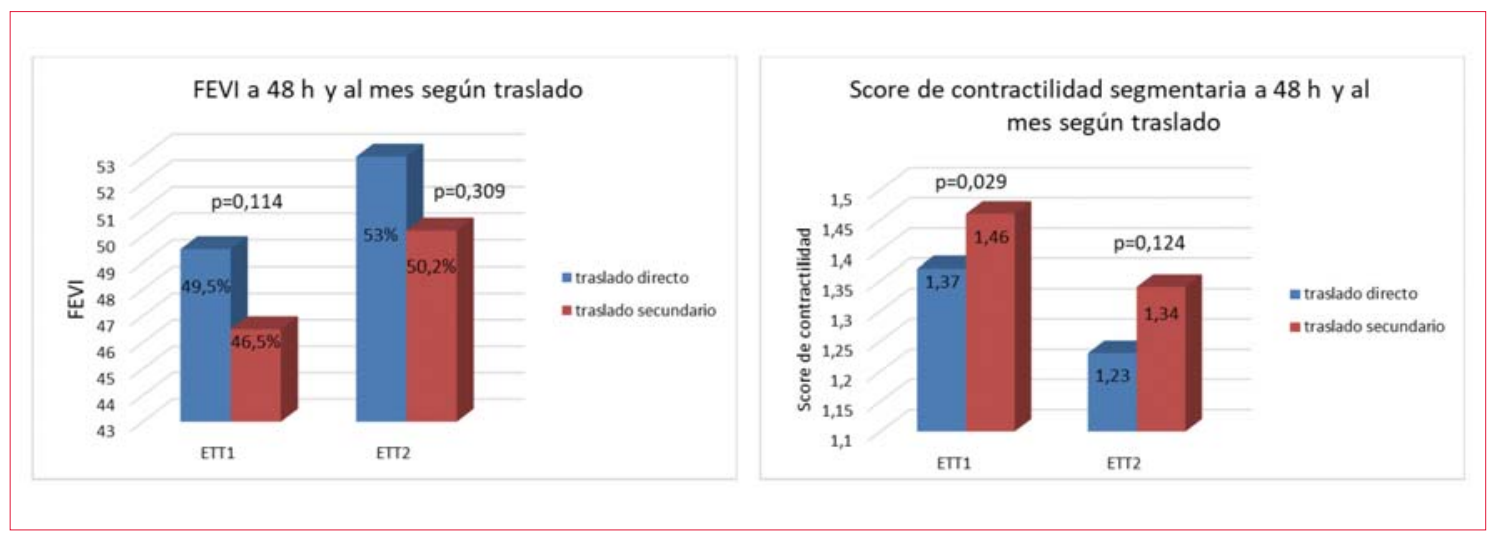

Figura 3. Análisis ecocardiográfico de la FEVI y score de contractilidad inicial y al mes por grupos. ETT 1: ecocardiograma transtorácico a las 48 horas. ETT 2: ecocardiograma transtorácico al mes.

se registró una diferencia significativa $(\mathrm{p}=0,012)$ en el score de contractilidad inicial, con un valor de $1,67 \pm 0,34$ en los pacientes que presentaron ECAM y de 1,40 $\pm 0,34$ para los pacientes libres de eventos. Al mes, la FEVI fue de $42,3 \pm 6,3 \%$ en los que tuvieron ECAM y de $51,8 \pm 9,7 \%$ en los libres de eventos $(p=0,031)$. El score de contractilidad al mes fue de $1,55 \pm 0,29$ y $1,28 \pm 0,29$ respectivamente $(p=0,065)$.

\section{Discusión}

El presente artículo evidencia que aún existe una importante demora en el acceso a la reperfusión del IAMCST, con una mediana de $300 \mathrm{~min}$. El registro uruguayo RENATIA (2006)(17) reportó una mediana de $250 \mathrm{~min}$, aunque solo incluyó a pacientes de hasta 12 horas de evolución.

Estos tiempos prolongados concuerdan con datos del US National Cardiovascular Registry de Chakrabarti obtenidos durante 2005 y $2006^{(18)}$, en que solo 9,9\% de los IAMCST cumplían con los tiempos recomendados por las guías.

Analizando los tiempos observamos que $45,3 \%$ del retardo corresponde al tiempo desde el inicio del dolor al PCM. Esto podría explicarse por una demora del paciente en consultar o por una dificultad en el acceso al sistema de salud, lo que podría corregirse con políticas educativas y mejoras del sistema que faciliten el acceso a la consulta inicial. Por otro lado, el $54,7 \%$ del tiempo de demora restante corresponde a demoras propias del sistema de salud, es decir, del tiempo PCM-puerta sumado al tiempo puerta-balón. En nuestro grupo de pacientes, el traslado directo desde domicilio a un centro con hemodinamia se asoció con una disminución significativa del tiempo isquémico en $43 \%$ y del tiempo PCM-balón en 58\% (disminuyendo así el tiempo PCM-puerta y el tiempo puerta-balón).

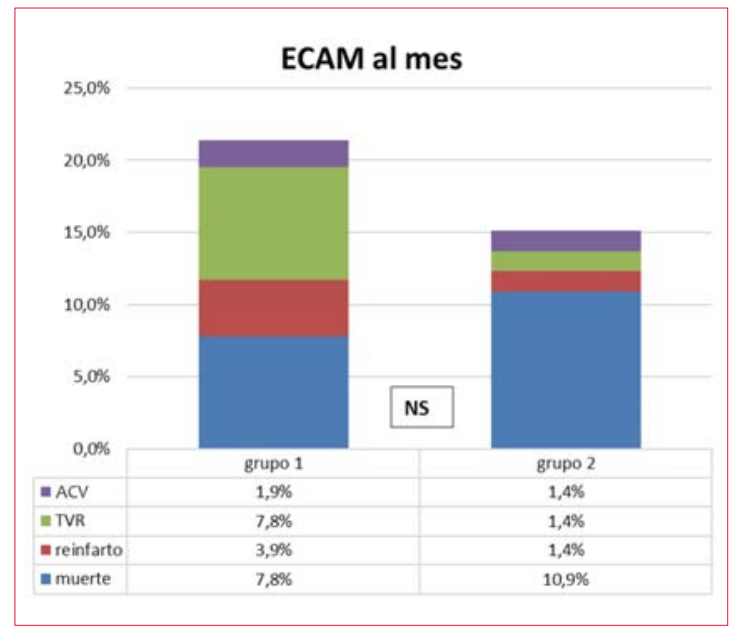

Figura 4. Tabla de ECAM al mes sin diferencias entre grupos con su gráfico correspondiente. Grupo 1 con traslado directo. Grupo 2: con traslado secundario. ECAM: eventos cardíacos adversos mayores. TVR: revascularización del vaso tratado.

Existe amplia bibliografía en relación con la disminución del tiempo de reperfusión asociada al traslado directo desde domicilio en el IAMCST. Los estudios de Dorsch y colaboradores ${ }^{(15)}$, con 387 pacientes en el Reino Unido; Le May y colaboradores ${ }^{(19)}$, con 344 pacientes en Ottawa; Pedersen y colaboradores ${ }^{(20)}$, con 1.334 pacientes en Dinamarca; Dieker ${ }^{(21)}$, con 581 pacientes en Holanda, y Estévez Loureiro y colaboradores ${ }^{(22)}$, con 1.194 pacientes en España, demostraron todos una disminución de la media del tiempo puerta-balón y tiempo isquémico total en los pacientes con traslado directo a sala de hemodinamia.

Encontramos una disminución del tiempo desde el inicio del dolor al PCM en el grupo de traslado directo, al igual que en el trabajo presentado en 2002 por Canto y colaboradores ${ }^{(23)}$. Es posible que esta diferencia esté vinculada a que los pacientes con traslado directo se hayan presentado con síntomas más típicos, que motiven una consulta temprana y una rápida res- 
puesta de los servicios de emergencia móvil, aspectos que no fueron analizados en nuestro trabajo.

En nuestra experiencia, los pacientes que fueron trasladados directamente desde domicilio a centro con hemodinamia fueron los únicos que presentaron una media de PCM-balón por debajo de los 120 minutos exigidos por las guías de práctica clínica, a diferencia de aquellos que consultaron por sus medios o fueron inicialmente trasladados hacia centros sin hemodinamia.

Los estudios de Bradley y colaboradores ${ }^{(24)}$ en 2005 y de Krumholz y colaboradores ${ }^{(25)}$ en 2008 evaluaron que la realización de un electrocardiograma prehospitalario permitía la inmediata activación del laboratorio y el equipo de hemodinamia, con una disminución del tiempo puerta-balón y un mejor cumplimiento de lo estipulado en las guías.

Los resultados de nuestro estudio, que concuerdan con datos internacionales, nos llevan a considerar que la implementación de medidas específicas como la activación prehospitalaria de los equipos de hemodinamia y el traslado directo del paciente a centros con sala de intervencionismo, evitando el pasaje por servicios de emergencia, contribuyen a reducir los tiempos de reperfusión.

Existe evidencia consistente sobre la relación entre el tiempo de reperfusión (farmacológica o intervencionista) y la mortalidad en el IAMCST. El Fibrinolitic Therapy Trialist (FTT) ${ }^{(26)}$, que analizó los estudios que utilizaron fibrinolíticos versus placebo en más de 1.000 pacientes con IAMCST, demostró la asociación entre el tiempo de demora en el tratamiento y la mortalidad. Resultados similares muestran Boersma y colaboradores ${ }^{(27)}$ en un metaanálisis publicado en 1996. Terkelsen y colaboradores $^{(28)}$ demostraron en 2010 un aumento en la mortalidad vinculado a la demora del sistema en lograr la reperfusión. Por otro lado, De Luca y colaboradores $^{(29)}$ concluyen que por cada 30 minutos de retardo en la ATC existe un aumento de 7,5\% en la mortalidad del IAMcST. Vinculado al tiempo de demora del sistema, Steg y colaboradores ${ }^{(30)}$, al igual que Pedersen y colaboradores ${ }^{(20)}$, demostraron que el pasaje del paciente por la emergencia se asocia a un peor pronóstico. Estévez-Loureiro y colaboradores $^{(22)}$ demuestran, mediante un análisis multivariado, que la aplicación sistemática del traslado directo fue un predictor pronóstico independiente. De igual modo, en el trabajo de Le May y colaboradores $^{(19)}$, la mortalidad a 180 días fue más del doble ( $11 \%$ vs $5 \%$ ) para los que tuvieron traslado a un centro sin hemodinamia.

Si bien en nuestro trabajo se observa una disminución de los tiempos vinculada al traslado directo desde domicilio a sala de hemodinamia, no se alcan- za una diferencia estadísticamente significativa de la mortalidad ni otros eventos clínicos, lo que podría estar vinculado al bajo número de pacientes incluidos.

Respecto al análisis ecocardiográfico, observamos que si bien el estudio no tuvo la potencia suficiente para demostrar diferencias significativas de la FEVI entre ambos grupos, sí evidenció un score de contractilidad significativamente mejor en el ETT inicial en el grupo con traslado directo. Un análisis post-hoc del estudio de Van't Hof ${ }^{(31)}$ evidenció que los pacientes con traslado directo a sala de hemodinamia tenían menos disfunción sistólica en comparación con los que eran valorados en emergencia. Nuestros hallazgos confirman que aquellos que tuvieron ECAM tenían peor score de contractilidad inicial y peor FEVI inicial y al mes.

La iniciativa Stent for Life (SFL) es un proyecto europeo iniciado en 2008 con el objetivo de conseguir que la mayoría de los pacientes con IAMCST tengan un rápido acceso a la intervención coronaria percutánea primaria, en un intento por disminuir la morbilidad y la mortalidad. La European Association for Percutaneous Cardiovascular Interventions (EAPCI) realizó una encuesta para analizar el uso de tratamientos de reperfusión en Europa, sugiriendo que el traslado primario de los pacientes con IAMCST debe evitar el paso por el hospital más próximo sin hemodinamia, así como por el servicio de urgencias o la unidad de cuidados intensivos del centro de hemodinamia. En consonancia, el paciente debe ser coordinado y trasladado directamente al laboratorio de hemodinamia, indicando el momento aproximado previsto para su llegada. Esta estrategia permite disminuir los plazos al mínimo, pudiendo alcanzarse el límite recomendado en las guías (<90 min) en la inmensa mayoría de los casos.

En el año 2010 se publicó en la Revista Uruguaya de Cardiología el Programa de infarto agudo de miocardio (PIAM), una iniciativa en vías de reformulación que aún no se ha puesto en práctica. Este programa tiene como objetivo asegurar el acceso de la terapia de reperfusión a todos los pacientes con IAMCST, reducir los tiempos de implementación de la misma y disponer tratamientos individualizados de óptima calidad. Se plantea un algoritmo de manejo en donde el PCM es la base para el éxito de la estrategia de reperfusión. Desde ese momento se debe proceder para asegurar el menor tiempo de acceso a la terapéutica de reperfusión. Algunas de las medidas propuestas consisten en la educación acerca del IAMCST y su manejo terapéutico, el apoyo en la implementación de la trombolisis y la puesta en marcha de un sistema de coordinación regional y un centro de apoyo telefónico para los médicos que se 
enfrentan al PCM. El PIAM también establece que en los pacientes valorados inicialmente por la UEM se proceda de inmediato al traslado directo a la sala de hemodinamia, siempre que el acceso a la misma cumpla con los tiempos estipulados por las guías, de lo contrario se deberá proceder al tratamiento trombolítico.

\section{Limitaciones}

Se realizó un estudio unicéntrico con un número pequeño de pacientes, lo que no permitió demostrar diferencias significativas en los ECAM y algunas variables ecocardiográficas asociadas al traslado directo desde domicilio. En el seguimiento, al mes hubo 16 pacientes que no concurrieron al control clínico y el mismo debió realizarse telefónicamente.

\section{Conclusión}

El traslado directo de los pacientes con IAMCST desde domicilio a un centro con servicio de hemodinamia se asoció con un menor tiempo isquémico total a expensas de un menor tiempo PCM-balón y con un mejor score de contractilidad segmentaria.

\section{Bibliografía}

1. Cannon CP, Gibson CM, Lambrew CT, Shoultz DA, Levy D, French WJ, et al. Relationship of symptom-onset-to-balloon time and door-to-balloon time with mortality in patients undergoing angioplasty for acute myocardial infarction. JAMA. 2000;283(22):2941-7.

2. MeNamara RL, Wang Y, Herrin J, Curtis JP, Bradley EH, Magid DJ, et al. Effect of door-to-balloon time on mortality in patients with ST-segment elevation myocardial infarction. J Am Coll Cardiol. 2006;47(11):2180-6.

3. Hasche ET, Fernandes C, Freedman SB, Jeremy RW. Relation between ischemia time, infarct size, and left ventricular function in humans. Circulation 1995;92(4):710-9.

4. Galli M, Marcassa C, Bolli R, Giannuzzi P, Temporelli PL, Imparato A, et al. Spontaneous delayed recovery of perfusion and contraction after the first 5 weeks after anterior infarction. Evidence for the presence of hibernating myocardium in the infarcted area. Circulation 1994;90(3):1386-97.

5. Bourdillon PD, Broderick TM, Williams ES, Davis C, Dillon JC, Armstrong WF, et al. Early recovery of regional left ventricular function after reperfusion in acute myocardial infarction assessed by serial two-dimensional echocardiography. Am J Cardiol 1989;63(11):641-6.

6. Keeley EC, Boura JA, Grines CL. Primary angio- plasty versus intravenous thrombolytic therapy for acute myocardial infarction: a quantitative review of 23 randomised trials. Lancet 2003;361(9351):13-20.

7. Grines CL, Browne KF, Marco J, Rothbaum D, StoneGW, O'KeefeJ, et al. A Comparison of Immediate Angioplasty with Thrombolytic Therapy for Acute Myocardial Infarction. The Primary Angioplasty in Myocardial Infarction Study Group. N Engl J Med 1993; 328(10): 673-9.

8. Widimský P, Groch L, Zelízko M, Aschermann M, Bednár F, Suryapranata H. Multicentre randomized trial comparing transport to primary angioplasty vs immediate thrombolysis vs combined strategy for patients with acute myocardial infarction presenting to a community hospital without a catheterization laboratory. The PRAGUE study. Eur Heart J 2000;21(10):823-31.

9. Widimský P, Budesínský T, Vorác D, Groch $\mathbf{L}$, Zelízko M, Aschermann M, et al. Long distance transport for primary angioplasty vs immediate thrombolysis in acute myocardial infarction. Final results of the randomized national multicentre trial-PRAGUE-2. Eur Heart J 2003;24(1):94-104.

10. Steg PG, James SK, Atar D, Badano LP, Blömstrom-Lundqvist C, Borger MA, et al. ESC Guidelines for the management of acute myocardial infarction in patients presenting with ST-segment elevation. Eur Heart J 2012;33(20):2569-619.

11. Angel Cequiera, Albert Ariza-Sole, Francisco J. Elola, Cristina Fernandez-Perez, Jose L. Bernal, Jose V. Segura et al. Impacto en la mortalidad de diferentes sistemas de asistencia en red para el tratamiento del infarto agudo de miocardio con elevación del segmento ST. La experiencia en España. Rev Esp Cardiol 2017;70(3):155-61.

12. Bradley EH, Curry LA, Webster TR, Mattera JA, Roumanis SA, Radford MJ, et al. Achieving rapid door-to-balloon times: how top hospitals improve complex clinical systems. Circulation 2006;113(8): 1079-85.

13. Dorsch MF, Greenwood JP, Priestley C, Somers K, Hague C, Blaxill JM, et al. Direct ambulance admission to the cardiac catheterization laboratory significantly reduces door-to-balloon times in primary percutaneous coronary intervention. Am Heart J 2008;155(6):1054-8.

14. Jacobs AK, Antman EM, Ellrodt G, Faxon DP, Gregory T, Mensah GA, et al. Recommendation to develop strategies to increase the number of ST-segment-elevation myocardial infarction patients with timely access to primary percutaneous coronary intervention. Circulation 2006;113(17):2152-63.

15. MeNamara RL, Herrin J, Bradley EH, Portnay EL, Curtis JP, Wang Y, et al. Hospital improvement in time to reperfusion in patients with acute 
myocardial infarction, 1999 to 2002. J Am Coll Cardiol 2006;47(1):45-51.

16. Comité de Hemodinamia de la Sociedad Uruguaya de Cardiología: Mayol J, Perna A, Albornoz H, et al. Angioplastia en el infarto agudo de miocardio dentro de las 24 horas de evolución. Análisis de la casuística uruguaya 2004-2012. Rev Urug Cardiol 2014; 29(1): 6-16

17. Comité de Hemodinamia de la Sociedad Uruguaya de Cardiología: Dres. C. Artucio, F. Buitrón, P. Díaz, T. Dieste, A. Durán, B. Erramún, et al. Registro Nacional del Tratamiento Intervencionista del Infarto Agudo de Miocardio en Uruguay (RENATIA). Rev Urug Cardiol 2006; 21(3): 218-23.

18. Chakrabarti A, Krumholz HM, Wang Y, Rumsfeld JS, Nallamothu BK. Time-to-reperfusion in patients undergoing interhospital transfer for primary percutaneous coronary intervention in the U.S an analysis of 2005 and 2006 data from the National Cardiovascular Data Registry. J Am Coll Cardiol 2008; 51(25): 2442-3

19. Le May MR, So DY, Dionne R, Glover CA, Froeschl MP, Wells GA, et al. A citywide protocol for primary PCI in STsegment elevation myocardial infarction. N Engl J Med 2008; 358(3): 231-40.

20. Pedersen SH, Galatius S, Hansen PR, Mogelvang R, Abildstrom SZ, Sørensen R, et al. Field triage reduces treatment delay and improves long-term clinical outcome in patients with acute ST-segment elevation myocardial infarction treated with primary percutaneous coronary intervention. $J$ Am Coll Cardiol 2009; 54(24): 2296-302.

21. Dieker HJ, Liem SS, El Aidi H, van Grunsven $P$, Aengevaeren WR, Brouwer MA, et al. Pre-hospital triage for primary angioplasty: direct referral to the intervention center versus interhospital transport. JACC Cardiovasc Interv 2010; 3(7) 705-11.

22. Estévez-Loureiro R, Calviño-Santos R, Vázquez-Rodríguez JM, Marzoa-Rivas R, Barge-Caballero E, Salgado-Fernández J, et al. Direct transfer of ST-elevation myocardial infarction patients for primary percutaneous coronary intervention from short and long transfer distances decreases temporal delays and improves short-term prognosis: the PROGALIAM Registry. Euro Intervention 2010; 6(3): 343-34.

23. Canto JG, Zalenski RJ, Ornato JP, Rogers WJ, Kiefe CI, Magid D, et al. Use of emergency medical services in acute myocardial infarction and subsequent quality of care: observations from the National
Registry of Myocardial Infarction 2. Circulation 2002; 106(24): 3018-23

24. Bradley EH, Roumanis SA, Radford MJ, Webster TR, McNamara RL, Mattera JA, et al. Achieving door-to-balloon times that meet quality guidelines: how do successful hospitals do it? J Am Coll Cardiol 2005; 46(7): 1236-41.

25. Krumholz HM, Bradley EH, Nallamothu BK, Ting HH, Batchelor WB, Kline-Rogers E, et al. A campaign to improve the timeliness of primary percutaneous coronary intervention: Door-to-Balloon: An Alliance for Quality. JACC Cardiovasc Interv 2008; 1(1): 97-104

26. Fibrinolytic Therapy Trialists' (FTT) Collaborative Group. Indications for fibrinolytic therapy in suspected acute myocardial infarction: collaborative overview of early mortality and major morbidity results from all randomised trials of more than 1000 patients. Lancet 1994; 343(8893): 311-22.

27. Boersma E, Maas AC, Deckers JW, Simoons ML. Early thrombolytic treatment in acute myocardial infarction: reappraisal of the golden hour. Lancet $1996 ; 348(9030): 771-7$.

28. Terkelsen CJ, Sørensen JT, Maeng M, Jensen LO, Tilsted HH, Trautner S, et al. System delay and mortality among patients with STEMI treated with primary percutaneous coronary intervention. JAMA 2010; 304: 763-71.

29. De Luca G, Suryapranata H, Ottervanger JP, Antman EM. Time delay to treatment and mortality in primary angioplasty for acute myocardial infarction: every minute of delay counts. Circulation 2004;109(10):1223-5.

30. Steg PG, Cambou JP, Goldstein P, Durand E, Sauval P, Kadri Z, et al. Bypassing the emergency room reduces delays and mortality in ST elevation myocardial infarction: the USIC 2000 registry. Heart 2006;92(10):1378-83.

31. Van't Hof AW, Rasoul S, van de Wetering H, Ernst N, Suryapranata H, Hoorntje JC, et al. Feasibility and benefit of prehospital diagnosis, triage, and therapy by paramedics only in patients who are candidates for primary angioplasty for acute myocardial infarction. Am Heart J 2006;151(6):1255. e1-1255.e5

32. Kaifoszovaa Z, Widimskyb P, Kristensen SD. La iniciativa Stent for Life en Europa. Rev Esp Cardiol Supl 2011;11(C):2-5.

33. Programa deI Infarto Agudo de Miocardio(PIAM) Rev Urug Cardiol 2010;25(3):217-25. 